\title{
An Unusual Lacrimal Gland Mass
}

\author{
Pankaj Gupta ${ }^{1}$, Savleen Kaur ${ }^{2}$, Zoramthara Zadeng ${ }^{3}$, Rakesh Vasishta ${ }^{4}$, Manpreet Singh ${ }^{5}$
}

\begin{abstract}
Aim and objective: The present report describes an atypical presentation of a lacrimal gland mass in a young male patient. Symptomatology favored a benign mass that was proven to be malignant on histopathology.

Background: Adenoid cystic carcinoma is the most common epithelial malignancy of the lacrimal gland. The usual presentation of adenoid cystic carcinoma is in either sex at about an average 40 years of age with range from 6 to 79 years. The patient usually complains of a superotemporal orbital mass with rapid progression usually within a year. Uncommonly, these masses may present with longer history as is described in present case report.

Case description: A 17-year-old male patient of Indian origin presented with a mass in the superotemporal part of the right orbit for the last 5 years and 6 months. A firm and smooth orbital mass, approximately $20 \mathrm{~mm} \times 15 \mathrm{~mm}$, was present in superotemporal orbit that was nontender and nonpulsatile. A clinical diagnosis of lacrimal gland pleomorphic adenoma was strongly suggested by the clinical as well as radiological features. The orbital mass was excised en bloc by a right transseptal anterior orbitotomy. Histopathological examination of the excised mass revealed features characteristic of lacrimal gland adenoid cystic carcinoma.

Conclusion: Lacrimal gland adenoid cystic carcinoma can simulate pleomorphic adenoma and can present with history over 5 years. Hence, it should always be kept as a clinical possibility in patients with the painless superotemporal orbital mass and early excision of such orbital masses for appropriate diagnosis and management is vital.

Clinical significance: The duration of 51/2 years at presentation in well-localized lacrimal gland adenoid cystic carcinoma is the longest period ever reported. The present article recommends keeping the differential diagnosis of adenoid cystic carcinoma in all patients with the clinically benign superotemporal orbital mass.
\end{abstract}

Keywords: Adenoid cystic carcinoma, Lacrimal gland, Orbital mass.

Clinical Rhinology An International Journal (2019): 10.5005/jp-journals-10013-1357

\section{BACKGROUND}

Lacrimal gland epithelial neoplasms are one of most intriguing primary orbital masses. The present report describes an atypical presentation of a lacrimal gland mass in a young male patient. Symptomatology favored a benign mass that was proven to be malignant on histopathology. The patient presented with 5.6 years' duration of symptoms, which to the best of our knowledge is the longest duration of presentation of well-localized painless lacrimal gland adenoid cystic carcinoma. A literature review of delayed presentations of lacrimal gland adenoid cystic carcinoma is also presented.

\section{Case Description}

A 17-year-old male patient of Indian origin presented with a mass in the superotemporal part of the right orbit for the last 5 years and 6 months. The mass was gradually progressive and showed no variation with posture or straining. The patient had no pain, redness, visual complaints, or any other systemic features. Examination revealed right-sided abaxial proptosis of $2 \mathrm{~mm}$ (Fig. 1A). A firm and smooth orbital mass, approximately $20 \mathrm{~mm} \times 15 \mathrm{~mm}$, was present in the superotemporal orbit that was nontender and nonpulsatile. The visual acuity was $6 / 6$ in both eyes. A clinical diagnosis of lacrimal gland pleomorphic adenoma was strongly suggested. The differential diagnosis included schwannoma, neurofibroma, and solitary fibrous tumor of superotemporal orbit. All these orbital masses are benign and can present as a slow-growing painless mass in the superotemporal part of the anterior orbit. A computed tomography (CT) scan of orbits revealed a localized homogeneous
1-3,5 Department of Ophthalmology, Postgraduate Institute of Medical Education and Research, Chandigarh, India

${ }^{4}$ Department of Histopathology, Postgraduate Institute of Medical Education and Research, Chandigarh, India

Corresponding Author: Pankaj Gupta, Department of Ophthalmology, Postgraduate Institute of Medical Education and Research, Chandigarh, India, Phone: +911722747837, e-mail: drpankajkgupta@gmail.com

How to cite this article: Gupta P, Kaur S, Zadeng Z, et al. An Unusual Lacrimal Gland Mass. Clin Rhinol An Int J 2019;12(2 and 3):49-51.

Source of support: Nil

Conflict of interest: None

hyperdense right superotemporal orbital mass (Fig. 1B) with bony fossa formation but showed no bone erosion. The orbital mass was excised en bloc by a right transseptal anterior orbitotomy. The mass was pinkish red, firm in consistency, and measured 34 $\times 30 \times 22 \mathrm{~mm}$ in largest dimensions (Fig. 1C). The intraoperative and postoperative periods were unremarkable. Histopathological examination of the excised mass revealed multiple lobules formed by malignant cells with pool of mucin forming the Swiss cheese pattern (Fig. 1D) characteristic of adenoid cystic carcinoma. The patient was planned for cytoreductive chemotherapy.

\section{Discussion}

Lacrimal gland epithelial neoplasms are perhaps the most intriguing primary orbital masses. Adenoid cystic carcinoma is the most common epithelial malignancy of the lacrimal gland. ${ }^{1}$ It 

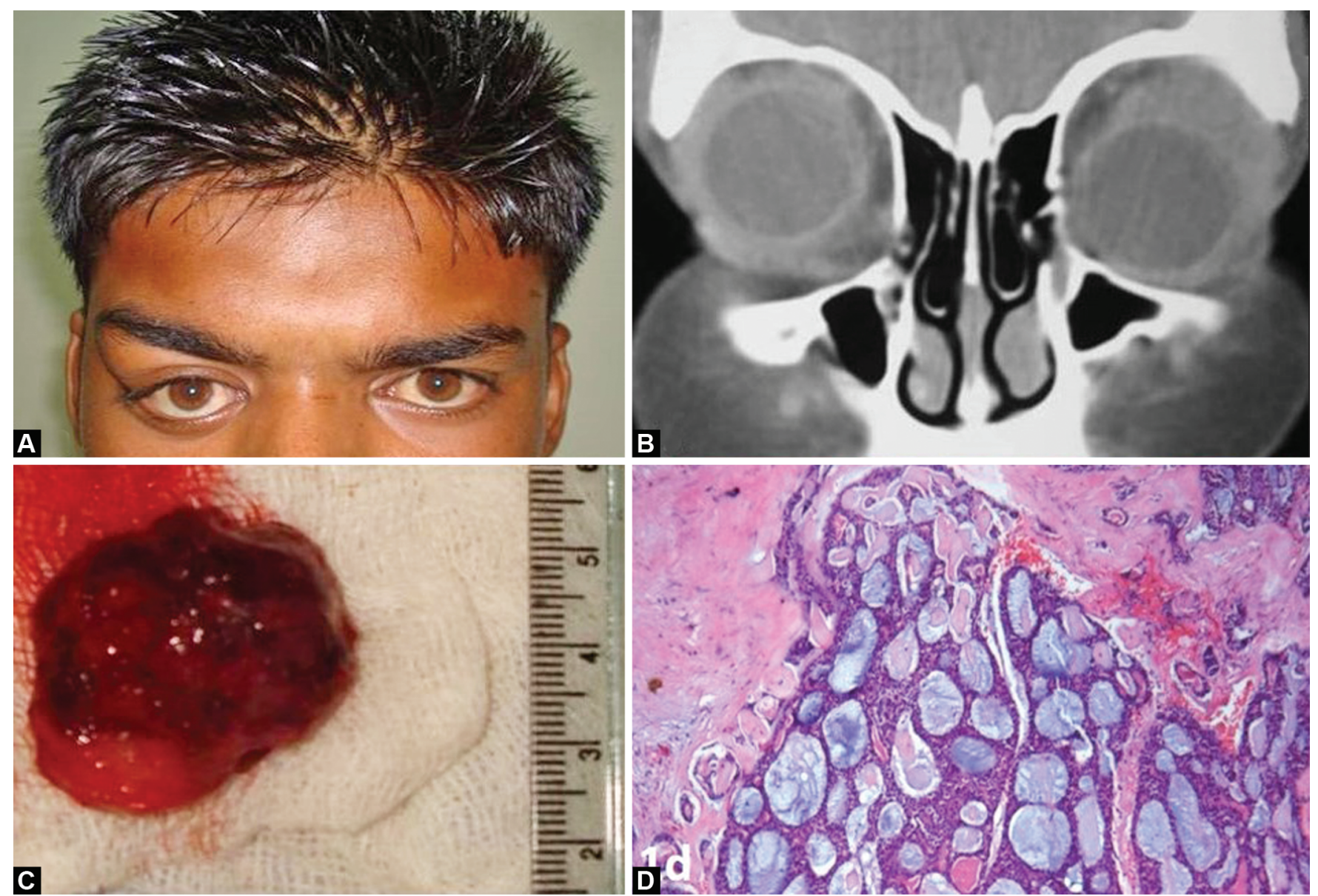

Figs 1A to D: (A) Clinical photograph of the patient who presented with a painless right superotemporal orbital mass; (B) Computed tomography revealed a homogeneous superotemporal orbital mass with no signs of bone erosion; (C) Photograph of the excised mass. The mass was pinkish red, firm in consistency, and measured $34 \times 30 \times 22 \mathrm{~mm}$ in largest dimensions; (D) Photograph of the histopathology review of the excised mass showing cribriform pattern of adenoid cystic carcinoma; stained with hematoxylin and eosin as viewed under 10× magnification

accounts for nearly $1.1 \%$ of all disorders involving the orbit ${ }^{2}$ and $63 \%$ of all primary epithelial malignant lacrimal gland masses. ${ }^{1}$ The usual presentation of adenoid cystic carcinoma is in either sex at about an average 40 years of age ${ }^{3}$ with range from 6 to 79 years. ${ }^{4}$ The patient complains of a superotemporal orbital mass with rapid progression usually within a year. ${ }^{5}$ However, in few instances the above is not adhered to. In the present patient, long history of a painless anterosuperior orbital mass with no features of malignancy favored a benign pathology. The differential diagnosis included schwannoma, neurofibroma, and solitary fibrous tumor involving the superotemporal part of the orbit. These were considered as the patient presented with signs and symptoms of a painless benign pathology. The radiological features revealed localized well-circumscribed mass with bony fossa formation that also favored a benign pathology. The final diagnosis, however, was adenoid cystic carcinoma. Wright et al. reported their experience with management of patients with adenoid cystic carcinoma. In their series, only one patient had symptoms for more than a year before presentation and all patients except for one had pain. ${ }^{3}$ Perez et al. in their series reported the mean time of presentation in adenoid cystic carcinoma to be 26.6 months. ${ }^{6}$ The patient with most extensive period of symptomatology prior to presentation in their series had periosteal involvement at presentation and was managed by wide orbital excision. ${ }^{6}$ Levartovsky et al. published a case study in 1993 about a female patient who had otherwise asymptomatic lacrimal gland mass for 3 years prior to presentation. ${ }^{7}$ The patient was kept under follow-up and the mass became painful after 1 year of presentation. The mass was then biopsied that revealed adenoid cystic carcinoma. The mass was excised and the patient given further required therapy. ${ }^{7}$ Wharton and O'Donnell reported a case of an incidentally discovered lacrimal gland mass that underwent excision and was confirmed to be adenoid cystic carcinoma. ${ }^{8}$ The authors concluded that adenoid cystic carcinoma may be discovered incidentally in occasional patient. ${ }^{8}$

This report represents the first case of localized painless adenoid cystic carcinoma of the lacrimal gland that presented after $51 / 2$ years of onset, which is the longest duration of presentation of well-localized painless adenoid cystic carcinoma to the best of our knowledge. With current practice, pleomorphic adenoma is usually diagnosed preoperatively based on clinical and radiological characteristics. Could it be in situ adenocarcinoma of the lacrimal gland that developed in a long-standing pleomorphic adenoma in the present case remains unanswered. The unusual history and findings in our patient highlight the clinical possibility of the rarer carcinomas with apparently benign course. 


\section{Conclusion}

Lacrimal gland adenoid cystic carcinoma can simulate pleomorphic adenoma and can present with history over 5 years. Hence, it should always be kept as a clinical possibility in patients with painless superotemporal orbital mass and early excision of such orbital masses for appropriate diagnosis and management is vital.

\section{Clinical Significance}

Lacrimal gland masses are among most intriguing primary orbital neoplasms. The present article describes a young male patient who presented with signs and symptoms of long-standing lacrimal gland pleomorphic adenoma that histopathology proved to be adenoid cystic carcinoma. The duration of 51/2 years at presentation in welllocalized lacrimal gland adenoid cystic carcinoma is the longest period ever reported. The present article recommends keeping the differential diagnosis of adenoid cystic carcinoma in all patients presenting with the clinically benign superotemporal orbital mass.

\section{ACKnOWLedgments}

Dr Suruchi Gupta and Dr Pooja Bansal, Senior Residents, Department of Ophthalmology, PGIMER, Chandigarh.

\section{References}

1. Shields JA, Shields CL, Epstein JA, et al. Primary epithelial malignancies of the lacrimal gland: the 2003 Ramon L Font LECTURE. Ophthalmic Plast Reconstr Surg 2004;20(1):10-21. DOI: 10.1097/01. IOP.0000103003.87842.BB.

2. Shields JA, Shields CL, Scartozzi R. Survey of 1264 orbital tumors and pseudotumors: The 2002 Montgomery lecture, part 1.Ophthalmology 2004;111(5):997-1008. DOI: 10.1016/j.ophtha.2003.01.002.

3. Wright JE, Rose GE, Garner A. Primary malignant neoplasms of the lacrimal gland. Br J Ophthalmol 1992;76(7):401-407. DOI: 10.1136/ bjo.76.7.401.

4. Kim YD. Lacrimal gland tumors. In: Karcioglu ZA, ed. Orbital tumors Diagnosis and management. New York USA: Springer; 2005. pp. 204-220.

5. Bartley G. Malignant lacrimal gland tumors. In: Rootman J, ed. Orbital disease. Florida USA: Taylor and Francis; 2005. pp. 107-115.

6. Perez DEC, Pires FR, Almeida OP, et al. Epithelial lacrimal gland tumors: a clinicopathological study of 18 cases. Otolaryngol Head Neck Surg 2006;134(2):321-325. DOI: 10.1016/j.otohns.2005.09.024.

7. Levartovsky S, Milstein A, Nissim F, et al. An unusual presentation of adenoid cystic carcinoma of lacrimal gland. Ophthal Plast Reconstr Surg 1993;9(1):47-50. DOI: 10.1097/00002341-199303000-00007.

8. Wharton JA, O'Donnell BA. Unusual presentations of pleomorphic adenoma and adenoid cystic carcinoma of the lacrimal gland. Aust N Z J Ophthalmol 1999;27(2):145-148. DOI: 10.1046/j.14401606.1999.00173.x. 